\title{
Developing a method for customized induction of flowering
}

\author{
Chin Chin Yeoh¹, Martin Balcerowicz', Rebecca Laurie², Richard Macknight ${ }^{2}$ and Joanna Putterill ${ }^{* *}$
}

\begin{abstract}
Background: The ability to induce flowering on demand is of significant biotechnological interest. FT protein has been recently identified as an important component of the mobile flowering hormone, florigen, whose function is conserved across the plant kingdom. We therefore focused on manipulation of both endogenous and heterologous FT genes to develop a floral induction system where flowering would be inhibited until it was induced on demand. The concept was tested in the model plant Arabidopsis thaliana (Arabidopsis).

Results: Our starting point was plants with strongly delayed flowering due to silencing of $F T$ with an artificial microRNA directed at FT (amiR-FT) [1]. First, we showed that constitutive expression of a heterologous FT gene (FTa1), from the model legume Medicago truncatula, (Medicago) was able to rescue the amiR-FT late-flowering phenotype. In order to induce flowering in a controlled way, the FTal gene was then expressed under the control of an alcohol-inducible promoter in the late flowering amiR-FT plants. Upon exposure to ethanol, FTa1 was rapidly up regulated and this resulted in the synchronous induction of flowering.

Conclusions: We have thus demonstrated a controlled-inducible flowering system using a novel combination of endogenous and heterologous FT genes. The universal florigenic nature of FT suggests that this type of system should be applicable to crops of economic value where flowering control is desirable.
\end{abstract}

\section{Background}

Flowering time is an important plant breeding target [reviewed by [2]]. The time at which flowering occurs affects the ensuing success of flower, seed and fruit development, ease of harvest and marketing. In addition, since flowering of vegetative crops and forages can be negatively correlated with yield and nutritive quality, the ability to delay flowering in such plants would be advantageous. Therefore, our goal is to develop molecular-genetic tools for customization of flowering in economically-important plants.

Plants use a combination of environmental and developmental cues to trigger flowering. The genetic networks that are involved in perception and response to these floral signals is best worked out in the model plant Arabidopsis thaliana (Arabidopsis) [3-8]. Many of the flowering time pathways ultimately converge on a set of genes called floral integrators, which includes

\footnotetext{
* Correspondence: J.Putterill@auckland.ac.nz

'Plant Molecular Sciences, School of Biological Sciences, University of

Auckland, Private Bag 92019, Auckland, New Zealand

Full list of author information is available at the end of the article
}

FLOWERING LOCUS T (FT) and a closely related and partially redundant homologue TWIN SISTER OF FT (TSF) [9-12]. FT strongly activates flowering in Arabidopsis and mutants lacking a functional $F T$ gene flower very late, while transgenic plants over expressing $F T$ flower much earlier than wild type plants $[9,10]$.

$F T$ is the primary target of several flowering time pathways. These include the long day pathway which promotes flowering in response to long day photoperiods via CONSTANS (CO) mediated up regulation of $F T$, and the vernalisation and autonomous pathways that function to down regulate the flowering repressor FLOWERING LOCUS C (FLC) thereby alleviating FLC's repression of $F T$ [reviewed by [6,7]]. $F T$ transcript is expressed in the leaf vasculature where FT protein is produced and moves via the phloem to the shoot tip [1,13-15]. In the shoot apical meristem, FT partners with FD, a bZIP transcription factor and switches on genes, such as APETALA1, to initiate floral development $[16,17]$. Thus, FT protein functions as an important component of the mobile flowering hormone, florigen [see reviews by $[6,8]]$. 
Older physiological studies demonstrated the universal nature of florigen in plants [reviewed by [18]]. Consistent with this, orthologues of the Arabidopsis FT gene are widespread in the plant kingdom (Figure 1a, b) and promote flowering even in plants with different day length requirements to Arabidopsis. For example, the tomato FT orthologue SINGLE FLOWER TRUSS (SFT) promotes flowering in day neutral tomato [19] and two rice FT orthologues, Heading date $3 a(H d 3 a)$ and RICE FLOWERING LOCUS T 1 (RFT1) promote flowering in rice, a short-day plant [20,21]. Like Arabidopsis FT, the $S F T, H d 3 a$ and RFT1 genes encode a graft transmissible floral signal $[19,22,23]$. In addition, over expression of FT orthologues such as SFT can promote flowering in heterologous transgenic plants [19]. Thus, despite differences in the upstream signaling pathways in different plants, the induction of FT expression in leaves and its movement to the apex where it triggers flowering appears to be conserved.

Genetic engineering of flowering time in many cultivated species has been achieved by manipulation of floral repressors or floral promoters, including the over expression or inducible expression of FT to accelerate flowering in transgenic plants $[2,24,25]$. Because of the conservation of FT function across the plant kingdom, we have focused on developing a controlled-inducible flowering system that manipulates endogenous and heterologous FT genes. This utilizes the induction of a heterologous $F T$ gene to trigger flowering in a background in which flowering has been inhibited using an artifical microRNA that targets the endogenous FT gene. The system is based on the idea that artificial microRNAs can be designed to specifically down regulate an endogenous gene, but should not affect the expression of a sufficiently divergent functional orthologue from another species. Here, we report on the development of this system for inducing flowering on demand and demonstrate its utility in Arabidopsis.

\section{Results and Discussion}

\section{Selecting a heterologous FT gene to overcome the late} flowering phenotype of amiR-FT Arabidopsis plants

Our starting point was a late flowering transgenic line of Arabidopsis in which flowering was inhibited by the expression of an artificial micro RNA directed against the $F T$ gene $(a m i R-F T)$ in the phloem companion cells $[1,26]$. This amiR-FT pairs with $F T$ transcript in the companion cells of the phloem and stimulates its degradation leading to gene silencing [26]. The amiR-FT sequence is complementary to bases 234 to 254 of the FT coding sequence (with one mismatch) (Figure 1c).

To overcome the late flowering phenotype of the amiR-FT plants, an FT orthologue was needed that would not be targeted by the amiR-FT, but could function to promote flowering in Arabidopsis. Since, in a related project we are investigating the role of $F T$ genes in flowering regulation in the model legume Medicago truncatula (Medicago), we investigated whether a Medicago FT gene might be a suitable heterologous candidate. There are five FT genes in Medicago; the partial genomic DNA sequences of three FT genes (FTa1, FTa2 and FTc) were described previously [27], while our database mining revealed full length sequences for these genes and two more, FTb1 and FTb2 (see also [28,29]). FTa1 is predicted to be the most closely related protein to FT with $71 \%$ identity. FTa2, FTb1 and FTb2 share at least $64 \%$ identity with FT, while FTc is slightly less similar at $61.7 \%$ identity to FT. Two key residues that are important for FT function in Arabidopsis [30,31] are present in four of the predicted Medicago FT proteins (FTa1, FTa2, FTb1 and FTb2) (Figure 1a). The fifth predicted protein, FTc, has the conserved tyrosine residue, but the conserved glutamine in FT is replaced by a histidine (Figure 1a).

Phylogenetic analysis of FT and the related TERMINAL FLOWER1 proteins, that repress flowering, shows that FTa1 and FTa2 form a sister clade to FT/TSF) (Figure 1b). However, although FTa1 shares the highest identity with Arabidopsis FT, all of the FT proteins are as closely related to FT as the known functional FT orthologues, Hd3a and RFT1 from rice $[20,21]$ and SFT from tomato [19].

To test if the Medicago FTa1 gene was likely to be targeted by the $a m i R-F T$, it was aligned with the $a m i R$ FT sequence. No significant similarity was identified using BLAST nucleotide searches. The best alignment that could be made using the MultAlin program is shown in Figure 1c. The number of mismatches in this alignment is 9 over the 21 base sequence, with 5 of these in the 5' region (from bases 2-12), which are important for efficient target-transcript down regulation [26]. This indicated that the FTa1 transcript was unlikely to pair with the amiR-FT and be degraded.

\section{Overexpression of $F T a 1$ rescues the late flowering phenotype of amiR-FT transgenic plants}

Our first aim was to over express FTa1 in the amiR-FT plants to test if this would rescue the late flowering phenotype. An expression construct with FTa1 fused to the 35 S promoter (35S::FTa1) was generated. Transformed T1 plants were selected, grown in flowering-inductive long day conditions (LD, $16 \mathrm{~h}$ light, $8 \mathrm{~h}$ dark) and their flowering time scored (Figure 2a).

Analysis of the leaf number at flowering showed that the majority of the 35S::FTa1 T1 plants (19/22) flowered considerably earlier than $a m i R-F T$ plants and wild type control plants, Columbia (Col) (Figure 2a). This result shows that over expression of FTa1 promotes flowering 
(a)
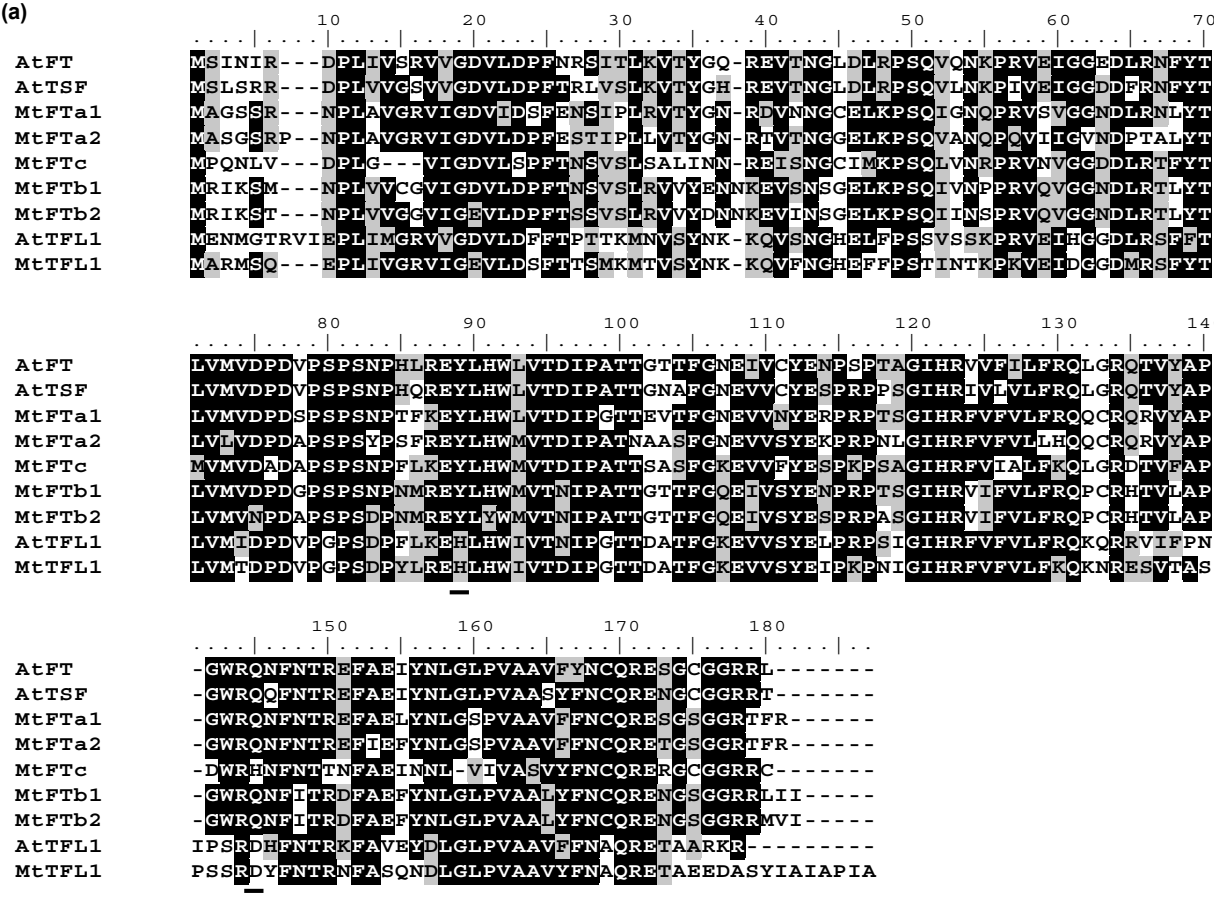

(b)

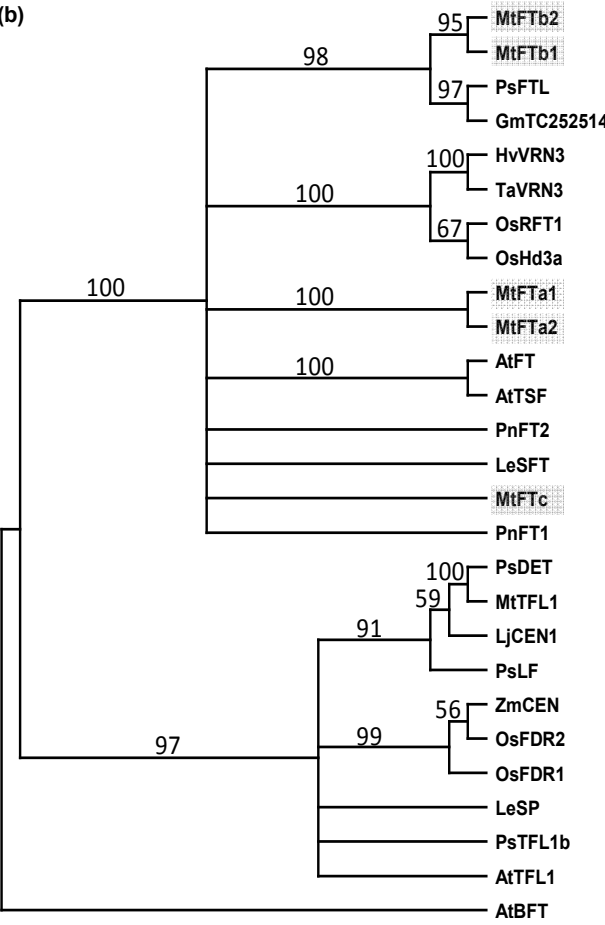

(c)

234

254

AtFT

+

, CAACCCUCACCUCCGAGAAUA....3

|||| ||||||||||||||||

amiR-FT $\quad 3{ }^{\prime}$...GUUGUGAGUGGAGGCUCUUAU....5'

332

352

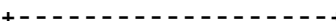

MtFTa1 5'...CACGACCCACUUCAGGGAUCC.... ${ }^{\prime}$

|| || ||| || | |

amiR-FT 3'...GUUGUGAGUGGAGGCUCUUAU...5'

Figure 1 Protein and mRNA sequence alignments and phylogenetic analysis of FT and TFL1 proteins. a) Alignment of selected full-length FT and TFL1 proteins from Medicago and Arabidopsis. Background black shading indicates identical amino acids shared in 50\% or more sequences, gray indicates similar amino acids shared in 50\% or more sequences. Black underlining marks key functional amino acid residues in FT (see text for details). b) Neighbour joining tree of selected FT and TFL1 full length proteins from 11 different plant species. The tree was rooted on AtBFT. Bootstrap values from 1000 replications are shown as a percentage above each branch. The Medicago proteins are highlighted with a gray box. c) Alignment of the sequence of the amiR-FT to Arabidopsis FT transcript (above) and to Medicago FTa1 mRNA (below). At Arabidopsis thaliana; Gm Glycine max. Hv Hordeum vulgare; Le Lycopersicon esculentum now Solanum lycopersicum; Lj Lotus japonicus, Mt Medicago truncatula Os Oryza sativa, rice, Pn Pharbitis nil, Ps Pisum sativum, Ta Triticum aestivum, Zm Zea mays. Gene identifiers (At) or Accession numbers are listed in the methods. 
by scoring the total number of leaves (rosette + cauline) at flowering, unless otherwise specified. The data is presented as mean +/-SE. Nineteen out of 22 of the 35S::FTa1 T1 transgenic plants flowered earlier than wild-type Columbia (Col) plants; the mean flowering time of these early flowering plants is shown. In the SD experiments, the control Col and amiR-FT plants had not yet flowered by 63 days after sowing, at which time the experiment was halted. The leaf number produced by the plants by this time is shown; these bars in the graph are marked with a dashed line b) FTa1 transcript accumulation in 10- day-old T3 transgenic lines and control plants in LD was measured using qRT-PCR. Relative transcript abundance of FTa1 $12 \mathrm{~h}$ after lights on in LD conditions is shown with levels normalised to At2g32170 (mean +/- SD of 3 PCR replicates is shown). (c) FT transcript accumulation in 10- day-old T3 transgenic lines and control plants in LD was measured using qRTPCR. Relative transcript abundance of FT $12 \mathrm{~h}$ after lights on in LD conditions is shown with levels normalised to At2g32170 (mean +/SD of 3 PCR replicates is shown)

in Arabidopsis and fully rescues the late flowering phenotype of amiR-FT transgenic plants. Thus, FTa1 functions to promote flowering in Arabidopsis, but does not appear to be targeted by the amiR-FT.

Before constructing an inducible version of FTa1, we confirmed that the early flowering phenotype observed in the T1 generation plants was heritable and expressed in their progeny. Two independent, homozygous T3 transgenic lines with single locus insertions were selected. The T3 transgenic lines were grown in LD or short day (SD, $8 \mathrm{~h} \mathrm{~L} / 16 \mathrm{~h} \mathrm{D})$ conditions. The T3 lines flowered much more rapidly than the control amiR-FT plants in both conditions (Figure 2a). These results confirmed that the early flowering trait was heritable. In addition, they showed that $35 S:: F T a 1$ also promoted flowering in non-inductive SD conditions in which flowering of the control Col plants are delayed (Figure 2a).

Expression of the FTa1 transgene and endogenous FT in the T3 lines and control plants in LD was measured by qRT-PCR (Figure $2 \mathrm{~b}$ and $2 \mathrm{c}$ ). Since Arabidopsis FT has cyclical diurnal expression, we harvested tissue $12 \mathrm{~h}$ after dawn when FT levels are rising in wild type plants grown in LD conditions [32]. As expected, in the presence of the amiR-FT, FT levels were lower in both FTa1 transgenic lines and the amiRNA-FT line as compared to Col plants (Figure 2c). In contrast, the FTa1 transcripts were detected at high levels in the 35S::FTa1 lines (Figure 2b). This result confirmed that the FTa1 transgene was abundantly expressed in the T3 generation plants and this was consistent with their early flowering phenotype.

Ethanol induces synchronous flowering in amiR-FT plants with an alc::FTa1 construct

Since expression of FTa1 from the $35 \mathrm{~S}$ promoter res-

cued the late flowering phenotype of the amiRNA-FT
Figure 2 Overexpression of Medicago FTa1 rescues the late flowering phenotype of amiR-FT plants. AmiR-FT Arabidopsis transgenic plants were transformed with a 355.:FTal gene expression construct. a) Graph showing average total leaf number at flowering of T1 plants $(n=19)$, two independent T3 homozygous transgenic lines with the 35S:::FTa1 construct and control plants ( $n=$ 11-16). The flowering time of transformant and control Arabidopsis plants in long day (LD) or short day (SD) conditions was measured 
lines, we generated an alcohol-inducible version of FTa1 (alc::FTa1) [33]. AmiR-FT Arabidopsis transgenic plants were transformed with the alc::FTa1 gene expression construct and $19 \mathrm{~T} 1$ transformants were selected. The T2 seed from these alc::FTa1 lines were sown out in LD and exposed to ethanol vapour. Eighteen of these T2 families segregated plants that flowered at 20 leaves or less (data not shown) which is much earlier than the amiRNA-FT control plants (see Figure 2a). This result suggested that flowering in these lines was inducible by ethanol treatment.

To confirm this result, four independent homozygous alc::FTa1 T3 Lines (TG1 to TG4) with single copy insertions were selected. Plants grown in LD were exposed to dual ethanol vapour treatments in which10 day-old seedlings were treated with ethanol for $48 \mathrm{~h}$ and then exposed for a second time at day 17 for $24 \mathrm{~h}$ and their flowering time recorded (Figure 3). Three of the TG lines (TG1, 2 and 4) showed strong induction of flowering compared to TG plants grown in the absence of ethanol. Analysis of leaf number at flowering (Figure $3 \mathrm{a}$ ) shows that the TG plants flowered as early or earlier (TG1, $12.1+/$ - SE 0.2; TG2, $10.0+/$ - SE 0.3 or TG4, 14.2 +/- SE 0.6 leaves) than wild type Col plants (14.9+/- SE 0.4 leaves). One other line (TG3) showed a much weaker flowering promotion in response to ethanol $(32.6+/-2.9$ leaves $)$ compared to the $-\mathrm{EtOH}$ treatment $(52+/-\mathrm{SE} 1.3$ leaves $)$. In the absence of ethanol, the TG lines all flowered late (ranging from $40.3+/$ - SE 1.1 to $54.5+/$ - SE 1.1 leaves) and at a similar time to the amiR-FT line (46.1 +/- SE 1.0 leaves). There was no effect of ethanol on flowering of control Col or amiR-FT plants.

The ability of ethanol to induce alc::FTa1 expression was examined using qRT-PCR (Figure 4). Three out of the four TG lines (TG1, 2 and 4) showed good induction of FTa1 expression after exposure to $24 \mathrm{~h}$ ethanol while one (TG3) showed very little induction (Figure 4a). The very low FTa1 induction in TG3 correlated with the delayed flowering in this line (Figure $3 \mathrm{a}$ and 3b). However, while TG1, TG2 and TG4 plants flowered at about the same time after induction, the level of FTa1 expression was very different. Thus there was an absence of direct correlation between the level of FTa1 induction and flowering in the rapid flowering lines. In the untreated TG lines, FTa1 was expressed at much lower levels (Figure 4a), but was detectable by qRTPCR. However, clearly this was not at sufficient levels to overcome the late flowering phenotype conferred by $a m i R-F T$. As expected, ethanol treatment did not alter endogenous $F T$ accumulation which was expressed at lower levels in the transgenic lines than wild type Col (Figure 4b).

In order to investigate the kinetics of FTa1 transcript accumulation, we carried out an induction time course.
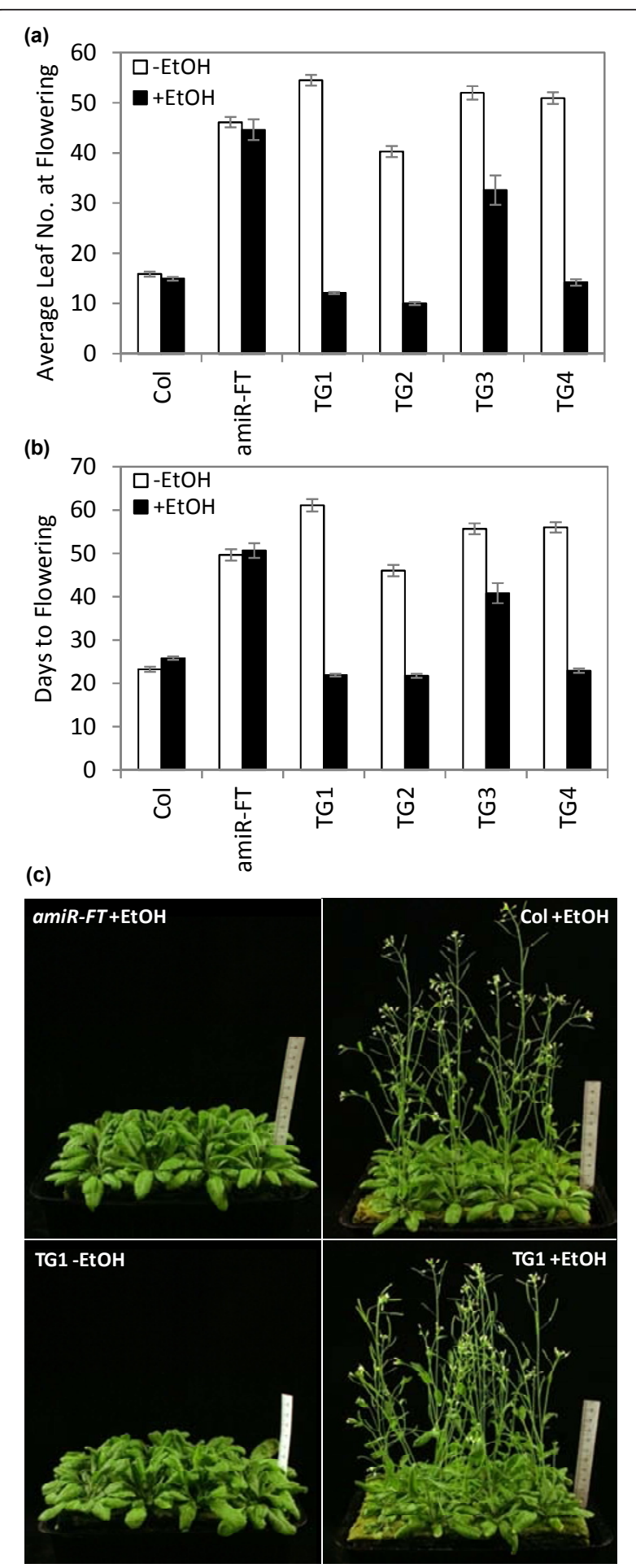

Figure 3 Ethanol vapour treatments induce flowering in amiRFT plants carrying an alcohol-inducible FTa1 gene expression cassette. AmiR-FT Arabidopsis transgenic plants were transformed with an alc:::FTa1 gene expression construct. Ten day-old Alc::FTa1 T3 generation transformants homozygous for single locus insertions (TG1 to TG4) or control plants, in LD conditions, were either exposed $(+\mathrm{ETOH})$, or not $(-\mathrm{ETOH})$, to ethanol vapour for $48 \mathrm{~h}$. Ethanol treatment was repeated 5 days later at day 17, for $24 \mathrm{~h}$. 
$\mathrm{n}=14-16$. a) Graph showing average total leaf number at flowering. Flowering time was measured by scoring the total number of leaves (rosette + cauline) at flowering. The data is presented as mean +/-SE. b) Graph showing days to flowering. The days to flowering after sowing were scored. The data is presented as mean +/-SE. c) Photographs of 39 day-old TG1 transgenic and control plants either exposed $(+\mathrm{ETOH})$, or not $(-\mathrm{ETOH})$, to ethanol vapour.

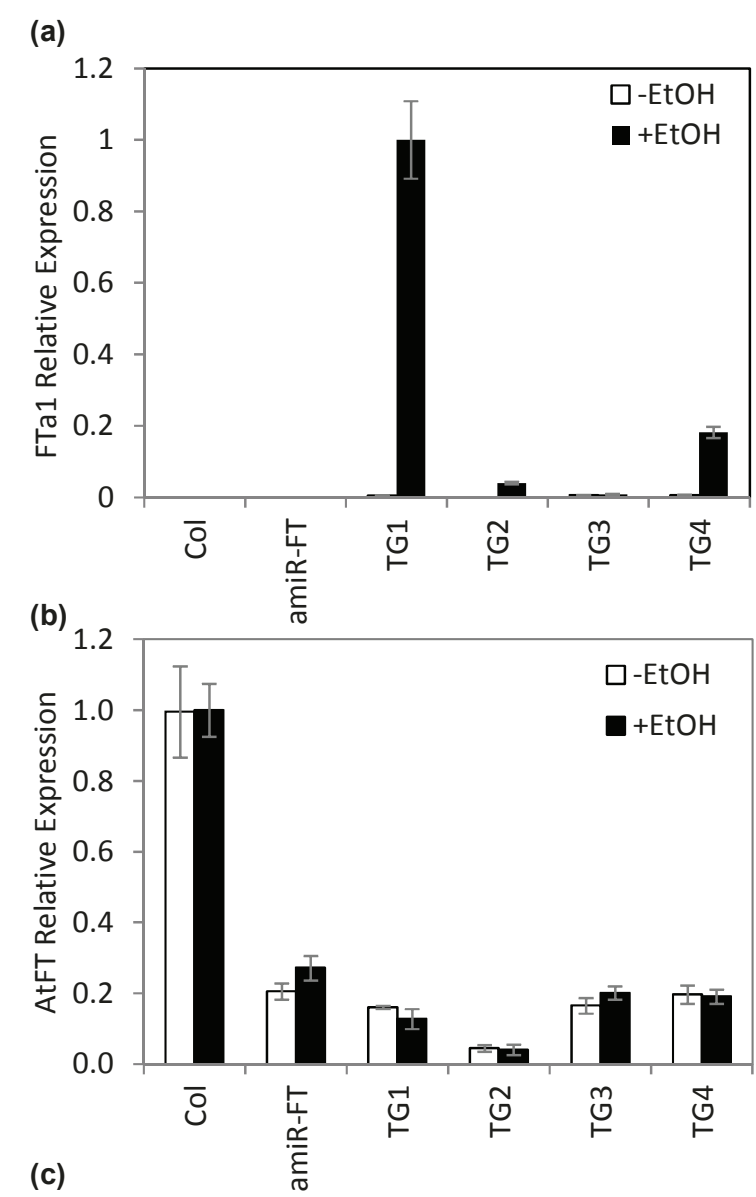

(c)

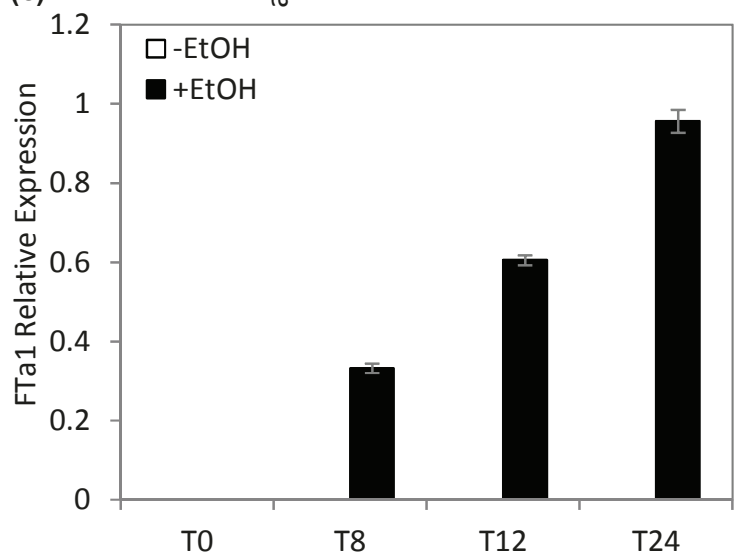

Figure 4 Induction of FTa1 expression by ethanol vapour. AlC: FTa1 T3 transformants or control plants in LD conditions were either exposed $(+\mathrm{ETOH})$, or not $(-\mathrm{ETOH})$, to ethanol vapour.
Transcript accumulation in the transgenic lines and control plants was measured using qRT-PCR with levels normalised to At2g32170. a) Relative transcript abundance of FTal after 10-day-old plants (TG1 to TG4 and controls) were exposed to ethanol vapour for $24 \mathrm{~h}$ (mean +/- SD of 3 PCR replicates is shown). Induction was started 4 $\mathrm{h}$ after dawn and plants were harvested 1 day later at the same time. b) Relative transcript abundance of endogenous Arabidopsis FT after ethanol induction as in (a) (mean +/- SD of 3 PCR replicates is shown). c) Time course of induction of FTal expression by ethanol vapour. Transcript accumulation was measured using qRTPCR with levels normalised to At2g32170. Time course of accumulation of FTa1 after 16 day-old TG1 plants were exposed to ethanol vapour for 8,12 or $24 \mathrm{~h}$ (mean + /- SD of qPCR on 2 biological replicates is shown). Induction was started at dawn.

FTa1 expression in TG1 plants in LD in response to ethanol vapour over $24 \mathrm{~h}$ was determined (Figure $4 \mathrm{c}$ ). Levels of FTa1 rose strongly within the first $8 \mathrm{~h}$ of ethanol exposure and continued to rise over the next $16 \mathrm{~h}$. No change in expression was observed in the untreated TG1 plants over the same time course. This rapid response to ethanol is consistent with previous reports of ethanol induction of alc:: reporter gene constructs [33].

These results indicated that ethanol vapour treatments were sufficient to induce FTa1 expression and synchronous early flowering and thus rescue the late flowering phenotype of the amiR-FT plants carrying the alc::FTa1 construct. In addition, the alc::FTa1 system gave tight control of the transition to flowering as the transgenic plants flowered late in the absence of ethanol.

\section{Manipulation of the timing of flowering}

Next we tested if the timing of flowering could be manipulated on demand by applying ethanol to plants of different ages. TG1 plants were grown in LD conditions and groups of 10,14 or 17 day-old plants were exposed to a single $48 \mathrm{~h}$ ethanol vapour treatment. We also repeated the dual ethanol treatment of 10-day- old plants as described above. In addition, we tested if flowering could be induced in SD conditions by exposure to ethanol. The number of days to flowering after the onset of each ethanol treatment was recorded for each plant (Figure 5).

In LD conditions, all ethanol treatments resulted in induction of flowering in the majority of plants (Figure $5 \mathrm{a}-\mathrm{d})$. Floral buds were first seen on the earliest-flowering plants 11 days after the onset of all the ethanol treatments. Thus these plants flowered 21 days, 25 days or 28 days after sowing. A control group of TG1 plants that were not exposed to ethanol were grown in parallel. The first of these plants (3/12 plants) started to flower 39 days after sowing in LD. Thus the alc::FTa1 plants could be induced to flower as early or earlier than wild type Col plants (flowered at $\sim 23$ days after sowing, 
treatment. Three out of 12 untreated TG1 plants had flowered by this time (the first visible flower buds were observed 39 days after sowing). b) Distribution of the time to flowering of 14-day-old plants exposed to ETOH for $48 \mathrm{~h}$. c) Distribution of the time to flowering of 17-day-old plants exposed to ETOH for 48 h. d) Distribution of the time to flowering of 10-day-old plants exposed to ETOH for $48 \mathrm{~h}$, followed by a further $24 \mathrm{~h}$ treatment when the plants were 17 days old. e) Distribution of the time to flowering of 20-day-old plants in SD conditions exposed to ETOH for $48 \mathrm{~h}$, followed by a further $24 \mathrm{~h}$ treatment when the plants were 27 days old. $n=24$. Seven plants had not yet flowered 30 days after the onset of ETOH treatment. Floral buds were first observed on 4/24 untreated TG1 plants at this time (50 days after sowing).

Figure $3 \mathrm{~b}$ ) or later than wild type, by varying the time at which ethanol is applied.

In LD, all of the 14 and 17 day-old-plants were induced to flower more rapidly than the untreated TG1 plants by a single exposure to ethanol (Figure 5b-c). However, the response of the 10-day-old plants to the single ethanol exposure was weaker (Figure 5a). While 10 of these plants were induced to flower by ethanol, the two remaining plants were not. One of these plants flowered 29 days after the start of the treatment (39 days after sowing), while the other still had not flowered by 30 days after the start of treatment (40 days after sowing). This was similar to the untreated TG1 controls.

The most effective of all the treatments in LD was the dual ethanol treatment of the 10-day-old TG1 plants in LD. This resulted in the most synchronous early flowering (Figure $5 \mathrm{~d}$ ). This indicated that there might be the need for more sustained expression of FTa1, particularly in younger plants in LD. This may be similar to wild type Arabidopsis plants, where endogenous FT is upregulated by exposing SD-grown plants to a single LD, but three LDs are required for commitment to flowering [34]. In addition, repeated induction of a heat-shock responsive $F T$ transgene was most effective at promoting the transition to flowering and ensuing normal flower development in transgenic poplar [24].

Flowering was also able to be induced in the majority of TG1 plants by ethanol in SD conditions (Figure 5e). Twenty day-old SD-grown plants were treated with ethanol for $48 \mathrm{~h}$ followed by a $24 \mathrm{~h}$ treatment 5 days later. Flower buds were seen 11 days after the onset of ethanol treatment on the earliest flowering plants (31 days after sowing). In LD, the earliest flowering plants also flowered 11 days after the initiation of ethanol treatment (Figure $5 \mathrm{a}-\mathrm{d}$ ). However, not all of the SD plants were induced to flower before the untreated TG1 plants. The latter began to flower from 50 days after sowing (4/24 plants). Seventeen ethanol-treated TG1 plants had flowered before the untreated TG1 controls began to flower, while seven had not (Figure 5e). This 
indicates that in SD there may be a need for more sustained induction of FTa1 or treatment of older plants to obtain floral induction in $100 \%$ of plants.

As controls for the induction experiment in SD, we also included ethanol- treated Col and amiR-FT plants. None of these plants had flowered by 63 days after sowing, at which time the experiment was halted. In addition, we grew a control alcohol-inducible transgenic line, the alc::GUS transgenic line [35]. This gene expression construct allows for ethanol-inducible expression of the $\beta$-GLUCURONIDASE (GUS) reporter gene. None of the alc::GUS plants, whether ethanol treated or untreated, had flowered by 63 days after sowing. Thus the early flowering phenotype of the TG1 plants in SD is due to ethanol induction of the alc::FTa1 geneexpression cassette.

\section{Conclusions}

Flowering time is a key trait in the breeding of crop and ornamental plants. Our aim was to create a universallyapplicable floral induction system that will allow flowering to be induced on demand. Here, we have described such a system and demonstrated its utility in the model plant Arabidopsis.

We showed that the expression of an FT orthologue from Medicago truncatula, FTa1, can overcome the delayed flowering of a transgenic Arabidopsis plants caused by expression of an artificial microRNA to the Arabidopsis FT gene, amiR-FT. Tight, inducible control of floral induction was engineered successfully using an alcohol-inducible version of the FTa1 gene. The timing of flowering could be manipulated by applying ethanol to plants of different ages, to give plants that flowered earlier or later than wild type. Endogenous Arabidopsis $F T$ remained low in the transgenic lines, consistent with ongoing silencing by the $a m i R-F T$. This indicates that the Medicago FTa1 is sufficiently different from Arabidopsis $F T$ to escape being targeted by the amiR-FT, yet has the ability to strongly promote flowering.

The advantage of the FT- based approach tested here in Arabidopsis is that because of the likely universal role of $F T$ in triggering of flowering, it should work in other plants. Inducing flowering when desired has many commercial uses [2]. For example, one application of our strategy might be to delay flowering in forage grasses during grazing to give consistent nutritive value and yield, but then later induce synchronous flowering for seed production. Other applications in crops could include inhibiting flowering, followed by inducing flowering to coincide with important market dates, or to avoid peak summer temperatures. The use of the alc inducible system should allow the system to be used in a field setting [reviewed by [36]]. Thus, our system has all the attributes required of a biotechnological floral induction system.

Although we have demonstrated the ability to overcome an artificial microRNA that targets a single $F T$ gene, in some cultivated species it might be important to down regulate multiple $F T$ genes to more effectively delay flowering. For example, Arabidopsis FT has the closely-related paralogue TSF and double $f t$ tsf mutants flower much later than the single mutants $[1,11]$. In rice, two key FT genes have an even stronger impact on flowering as plants with RNAi silencing of both genes had not flowered by 300 days after sowing [21]. Mathieu et al. (2007) demonstrated that it was possible to design an artificial microRNA that could target both the Arabidopsis FT and TSF mRNA simultaneously. Plants overexpressing this amiR construct phenocopied the late flowering of the $f t$ tsf double mutant. Therefore, the use of one or more artifical microRNAs should allow all the $F T$ family members involved in the induction of flowering of a particular species to be targeted. Care would then need to be taken to select an FT orthologue from another species that would be unaffected by the $\operatorname{amiR}(s)$.

In some cultivated species, natural variants exist that have strongly delayed flowering [2]. In most cases, the genetic reason for the delayed flowering has not been determined. However, it is likely that often this is caused by alterations to pathways upstream of $F T$ that prevent or delay the induction of FT. Thus, it is an attractive possibility that the late flowering of many natural variants could be utilized in our inducible flowering system so that the targeting of the endogenous FT genes using $a m i R$ would not be necessary and that flowering could be induced simply by using the alcohol-inducible FT gene.

In summary, we present proof-of-principle experiments that demonstrate a novel system for inducing flowering on demand, which should provide a biotechnological method for the customization of flowering of commercially important plants.

\section{Methods}

Database searches, sequence alignments and phylogenetic analysis

FT and TFL1 genes in Medicago truncatula (Medicago) were identified by tBLASTn searches (Basic Local Alignment Search Tool, BLAST) with Arabidopsis FT against Medicago genomic and EST sequences in the National Centre for Biotechnology Information (NCBI) database http://www.ncbi.nlm.nih.gov/blast and the DFCI Medicago Gene Index database http://compbio.dfci.harvard. edu/tgi/cgi-bin/tgi/gimain.pl?gudb=medicago. FT and TFL1 proteins from other species were obtained from GenBank. Protein sequence alignments were performed with ClustalW ( ${ }^{\odot} 2007$ Des Higgins, Julie Thompson, 
Toby Gibson) and in some cases manually adjusted using the BioEdit Sequence Alignment Editor $\left({ }^{\odot} 1997-\right.$ 2007 Tom Hall). BioEdit was also used to calculate percentage identity and percentage similarity between a pair of sequences after alignment. After alignment, boot strap analysis with 1000 replications was performed with SEQBOOT. The datasets were then subjected to distance matrix-based phylogenetic analysis using the programs PROTDIST and NEIGHBOR. CONSENSE was used to combine all datasets into one tree based on the majority rule consensus method which only includes groups that are present in more than $50 \%$ of the individual trees. All phylogenetic programs were distributed with the Phylogeny Inference Package (PHYLIP) 3.68 $\left({ }^{\odot} 1980-2008\right.$ University of Washington). Phylogenetic trees were displayed with TREEVIEW 1.6.6 ( ${ }^{\odot} 2000$ Roderic D.M. Page).

Gene identifiers or Accession numbers are AtFT At1g65480, AtBFT At5g62040, AtTFL1 At5g03840, AtTSF At4g20370, Gm TC252514, HvVRN3 TC168728, LeSFT AY186735, LeSP U84140, LjCEN AY423715, MtFTa1 HQ721813; MtFTa2 HQ721814, MtFTb1 HQ721815, MtFTb2 HQ721816, MtFTc HQ721817, MtTFL1 TC129531, OsFDR1 AF159883, OsFDR2 TC304905, OsHD3a TC315022, OsRFT1 TC315393, PnFT1 EU178859, PnFT2 EU178860, PsFT AY830923, PsDET AY340579, PsLF AY343326, PsTFL1b AY340580, TaVRN3 TC322000, ZmCEN TC388266.

Alignment of the mRNA sequence of the artificial microRNA to FT (amiR-FT) with the predicted mRNA of the FTa1 gene was done using the MultAlin program [37].

\section{Plant material, flowering time measurements and ethanol treatments}

All plant material used in this work was derived from the Arabidopsis thaliana L. Heynh accession Columbia (Col). The amiR-FT transgenic line (SUC2::amiR-FT; \#NW48_ 1-1) was described previously [1] as was the alc::GUS (AlcAGus) transgenic line [35]. Flowering time and gene expression analyses were carried out on plants grown under long-day conditions (LD, 16 h light/8 h dark) or short days (SD, $8 \mathrm{~h}$ light/16 h dark) in Percival growth cabinets in $\sim 150 \mu \mathrm{M} \mathrm{m}^{-2} \mathrm{~s}^{-1}$ cool white fluorescent light at $22^{\circ} \mathrm{C}$ in rockwool blocks moistened with hydroponics media [38] [without $\mathrm{Na}_{2} \mathrm{SiO}_{3}$ ]. Flowering time measurements were carried out by recording the total leaf number at the time of flowering and the days to flowering. Analysis of the flowering time of plants in the presence of ethanol was carried out by exposing plants to ethanol vapour provided by two microfuge tubes each containing $2 \mathrm{ml}$ of $100 \%$ ethanol placed at the opposite ends of a rockwool block. The plants and tubes were enclosed using a clear plastic lid (length $38 \mathrm{~cm}$, width $24 \mathrm{~cm}$, height $12 \mathrm{~cm}$ ) from a Stewart Unheated Propagator which was not airtight. The ethanol regimes used are described in the text. Analysis of gene expression in the presence of ethanol was carried out by exposing plants to ethanol vapour. The regimes used are described in the text.

\section{Plasmids and plant transformation}

A genomic clone with the coding region and introns of the FTa1 gene from Medicago truncatula were fused to the CaMV $35 S$ promoter (35S::FTa1) by recombination in the Gateway binary vector PK2GW7 [39], or to the $A l c A$ promoter (alc::FTa1) in a Gateway compatible alcR-alcA binary vector [[33], modified to be Gateway compatible by Lawrence Hobbie and Catherine PerrotRechenmann CNRS, Gif sur Yvette, unpublished]. Details of the cloning procedures can be obtained from the authors. The constructs were transformed into $a m i R-F T$ transgenic plants which were Basta resistant. The kanamycin-resistant T1 transformants were selected in vitro and rescued onto rockwool blocks. Independent homozygous, single copy, T3 lines were bred and used for further work. The presence and identity of the transgenes in the transformed lines were confirmed by PCR and DNA sequencing.

\section{RNA extraction, cDNA synthesis and qRT-PCR}

For gene expression experiments, RNA was extracted from 50 - $100 \mathrm{mg}$ of pooled plant tissue (total aerial parts) using the RNeasy ${ }^{\circledR}$ Plant Mini Kit (Qiagen). A TURBO DNase on-column treatment was carried out after RNA extraction (TURBO DNA-free ${ }^{\mathrm{TM}} \mathrm{Kit}$, Applied Biosystem). RNA was quantified using a NanoDrop ${ }^{\circledR} \mathrm{N}$ 1000 Spectrophotometer (NanoDrop Technologies Inc.). One microgram total RNA was transcribed into cDNA with Superscript III reverse transcriptase (Invitrogen) according to the manufacturer using a $(\mathrm{dT})_{17}$ primer (5'-GACTCGAGTCGACATCGATTTTTTTTTTTT TTTTT-3') [40]. As a control for potential genomic DNA contamination, the same procedure was carried out omitting the reverse transcriptase. To determine relative gene expression levels using quantitative Real Time PCR (qRT-PCR), $2 \mu \mathrm{l}$ of a 20 -fold diluted solution of cDNA was used in a total reaction volume of $10 \mu \mathrm{l}$ $1 \times$ SYBR $^{\circledR}$ Green PCR Master Mix (Applied Biosystems) with final primer concentrations of $0.5 \mu \mathrm{M}$. Each cDNA sample was analysed in triplicate PCR reactions, on a 7900 HT Sequence Detection system (Applied Biosystems). Relative gene expression levels were calculated using the $2^{-\Delta \Delta C T}$ method [41]. The gene expression experiments were repeated on independently grown plants and similar results were obtained. Primers used for quantification of gene expression levels were tested for amplification efficiency prior to use with a dilution series of an arbitrary cDNA sample. The following 
primer pairs were used for qRT-PCR; $F T$, 5'-CTGGAAC AACCTTTGGCA AT-3'and 5'-TACACTGTTTGCCTG CCAAG-3'; FTa1, 5' - GTAGCAGTAGGAATCCACT AG C-3' and 5' - ACACTCACTCTCGGTTGATTTCC3', At2g32170 [42], 5'-TGCTTTTTCATCGACACTGC3 ' and 5'-CCATATGTGTCCGCAAAATG-3'.

\section{Acknowledgements}

We thank Markus Schmid for his kind gift of the amiR-FT transgenic line (SUC2::amiR-FT; \#NW48_1-1) and Catherine Perrot-Rechenmann and Lawrence Hobbie for their kind gift of the Gateway-based alcA-alcR vector and the alc:::GUS transgenic line (AlCAGUS). We thank Hong Liu and Eric Liu for their technical assistance and Robert Schaffer, Karine David and Erika Varkonyi-Gasic for critical reading of the manuscript. We thank the New Zealand Foundation for Research Science and Technology for funding, contract C10X0704.

\section{Author details}

Plant Molecular Sciences, School of Biological Sciences, University of Auckland, Private Bag 92019, Auckland, New Zealand. ²Department of Biochemistry, University of Otago, PO Box 56, Dunedin 9054, New Zealand.

\section{Authors' contributions}

CY carried out flowering time and gene expression experiments and drew the figures, MB carried out database searches, alignments and phylogenetic analysis, drew figures and wrote some of the text, RL and RM provided unpublished materials and results, helped to conceive the study and write the manuscript. JP conceived of the study, supervised the overall project and wrote the manuscript. All authors read and approved the final manuscript.

\section{Received: 22 September 2010 Accepted: 11 April 2011}

Published: 11 April 2011

\section{References}

1. Mathieu J, Warthmann N, Kuttner F, Schmid M: Export of FT protein from phloem companion cells is sufficient for floral induction in Arabidopsis. Current Biology 2007, 17(12):1055-1060.

2. Jung C, Muller AE: Flowering time control and applications in plant breeding. Trends in Plant Science 2009, 14(10):563-573.

3. Putterill J, Laurie R, Macknight R: It's time to flower: the genetic control of flowering time. BioEssays 2004, 26:363-373.

4. Baurle I, Dean C: The timing of developmental transitions in plants. Cell 2006, 125(4):655-664.

5. Mouradov A, Cremer F, Coupland G: Control of flowering time: Interacting pathways as a basis for diversity. Plant Cell 2002, 14:S111-S130.

6. Turck F, Fornara F, Coupland G: Regulation and identity of florigen: FLOWERING LOCUS T moves center stage. Annual Review of Plant Biology 2008, 59:573-594.

7. Kim DH, Doyle MR, Sung S, Amasino RM: Vernalization: Winter and the Timing of Flowering in Plants. Annual Review of Cell and Developmental Biology 2009, 25:277-299.

8. Kobayashi Y, Weigel D: Move on up, it's time for change - mobile signals controlling photoperiod-dependent flowering. Genes \& Development 2007 21(19):2371-2384.

9. Kardailsky I, Shukla VK, Ahn JH, Dagenais N, Christensen SK, Nguyen JT, Chory J, Harrison MJ, Weigel D: Activation tagging of the floral inducer FT. Science 1999, 286:1962-1965.

10. Kobayashi $Y$, Kaya H, Goto K, Iwabuchi M, Araki T: A pair of related genes with antagonistic roles in mediating flowering signals. Science 1999, 286:1960-1962.

11. Yamaguchi A, Kobayashi Y, Goto K, Abe M, Araki T: TWIN SISTER OF FT (TSF) acts as a floral pathway integrator redundantly with FT. Plant and Cell Physiology 2005, 46(8):1175-1189.

12. Liu C, Thong ZH, Yu H: Coming into bloom: the specification of floral meristems. Development 2009, 136(20):3379-3391.

13. Corbesier L, Vincent C, Jang SH, Fornara F, Fan QZ, Searle I, Giakountis A, Farrona S, Gissot L, Turnbull C, et al: FT protein movement contributes to long-distance signaling in floral induction of Arabidopsis. Science 2007, 316(5827):1030-1033.

14. Jaeger KE, Wigge PA: FT protein acts as a long-range signal in Arabidopsis. Current Biology 2007, 17(12):1050-1054.

15. Takada S, Goto K: TERMINAL FLOWER2, a HETEROCHROMATIN PROTEIN1Like Protein of Arabidopsis, counteracts the activation of FLOWERING LOCUS $T$ by CONSTANS in the vascular tissues of leaves to regulate flowering time. Plant Cell 2003, 15:2856-2865.

16. Abe M, Kobayashi $Y$, Yamamoto $S$, Daimon $Y$, Yamaguchi A, Ikeda $Y$, Ichinoki H, Notaguchi M, Goto K, Araki T: FD, a bZIP protein mediating signals from the floral pathway integrator FT at the shoot apex. Science 2005, 309(5737):1052-1056

17. Wigge PA, Kim MC, Jaeger KE, Busch W, Schmid M, Lohmann JU, Weigel D: Integration of spatial and temporal information during floral induction in Arabidopsis. Science 2005, 309(5737):1056-1059.

18. Zeevaart JAD: Leaf-produced floral signals. Current Opinion in Plant Biology 2008, 11(5):541-547.

19. Lifschitz E, Eviatar T, Rozman A, Shalit A, Goldshmidt A, Amsellem Z Alvarez JP, Eshed $Y$ : The tomato FT ortholog triggers systemic signals that regulate growth and flowering and substitute for diverse environmental stimuli. Proceedings of the National Academy of Sciences of the United States of America 2006, 103(16):6398-6403.

20. Kojima S, Takahashi Y, Kobayashi Y, Monna L, Sasaki T, Araki T, Yano M: $H d 3 a$, a rice ortholog of the Arabidopsis FT gene, promotes transition to flowering downstream of $\mathrm{Hd} 1$ under short-day conditions. Plant and Cell Physiology 2002, 43(10):1096-1105.

21. Komiya R, Ikegami A, Tamaki S, Yokoi S, Shimamoto K: Hd3a and RFT1 are essential for flowering in rice. Development 2008, 135(4):767-774.

22. Tamaki S, Matsuo S, Wong HL, Yokoi S, Shimamoto $\mathrm{K}$ : Hd3a protein is a mobile flowering signal in rice. Science 2007, 316(5827):1033-1036.

23. Komiya R, Yokoi S, Shimamoto K: A gene network for long-day flowering activates RFT1 encoding a mobile flowering signal in rice. Development 2009, 136(20):3443-3450.

24. Zhang HL, Harry DE, Ma C, Yuceer C, Hsu CY, Vikram V, Shevchenko O, Etherington E, Strauss SH: Precocious flowering in trees: the FLOWERING LOCUS T gene as a research and breeding tool in Populus. Journal of Experimental Botany 2010, 61(10):2549-2560.

25. Kotoda $N$, Hayashi H, Suzuki M, Igarashi M, Hatsuyama $Y$, Kidou S, Igasaki T, Nishiguchi M, Yano K, Shimizu T, et al: Molecular Characterization of FLOWERING LOCUS T-Like Genes of Apple (Malus domestica Borkh). Plant and Cell Physiology 2010, 51(4):561-575.

26. Schwab R, Ossowski S, Riester M, Warthmann N, Weigel D: Highly specific gene silencing by artificial microRNAs in Arabidopsis. Plant Cell 2006, 18(5):1121-1133.

27. Hecht V, Foucher F, Ferrandiz C, Macknight R, Navarro C, Morin J, Vardy ME, Ellis N, Beltran JP, Rameau C, et al: Conservation of Arabidopsis flowering genes in model legumes. Plant Physiology 2005, 137(4):1420-1434.

28. Liew LC, Hecht V, Laurie RE, Knowles CL, Schoor JKV, Macknight RC, Weller JL: DIE NEUTRALIS and LATE BLOOMER 1 Contribute to Regulation of the Pea Circadian Clock. Plant Cell 2009, 21(10):3198-3211.

29. Hecht V, Laurie RE, Vander Schoor JK, Ridge S, Knowles CL, Liew LC, Sussmilch FC, Murfet IC, Macknight RC, Weller JL: The Pea GIGAS Gene Is a FLOWERING LOCUS T Homolog Necessary for Graft-Transmissible Specification of Flowering but Not for Responsiveness to Photoperiod. Plant Cell 23(1):147-161.

30. Ahn JH, Miller D, Winter VJ, Banfield MJ, Lee JH, Yoo SY, Henz SR, Brady RL, Weigel D: A divergent external loop confers antagonistic activity on floral regulators FT and TFL1. Embo Journal 2006, 25(3):605-614.

31. Hanzawa $Y$, Money T, Bradley D: A single amino acid converts a repressor to an activator of flowering. Proceedings of the National Academy of Sciences of the United States of America 2005, 102(21):7748-7753.

32. Suarez-Lopez P, Wheatley K, Robson F, Onouchi H, Valverde F, Coupland G: CONSTANS mediates between the circadian clock and the control of flowering in Arabidopsis. Nature 2001, 410(6832):1116-1120.

33. Roslan HA, Salter MG, Wood CD, White MRH, Croft KP, Robson F, Coupland G, Doonan J, Laufs P, Tomsett AB, et al: Characterization of the ethanol-inducible alc gene-expression system in Arabidopsis thaliana. Plant Journal 2001, 28(2):225-235.

34. Valverde F, Mouradov A, Soppe W, Ravenscroft D, Samach A, Coupland G: Photoreceptor Regulation of CONSTANS protein in photoperiodic flowering. Science 2004, 303:1003-1006. 
35. Braun N, Wyrzykowska J, Muller P, David K, Couch D, Perrot-Rechenmann C, Fleming AJ: Conditional Repression of AUXIN BINDING PROTEIN1 Reveals That It Coordinates Cell Division and Cell Expansion during Postembryonic Shoot Development in Arabidopsis and Tobacco. Plant Cell 2008, 20(10):2746-2762

36. Padidam M: Chemically regulated gene expression in plants. Current Opinion in Plant Biology 2003, 6(2):169-177.

37. Corpet F: Multiple sequence alignment with hierarchical clustering. Nucleic Acids Research 1988, 16:10881-10890.

38. Gibeaut DM, Hulett J, Cramer GR, Seemann JR: Maximal biomass of Arabidopsis thaliana using a simple, low-maintenance hydroponic method and favorable environmental conditions. Plant Physiology 1997, 115:317-319.

39. Karimi M, Inze D, Depicker A: Gateway vectors for Agrobacteriummediated plant transformation. Trends in Plant Science 2002, 7:193-195.

40. Frohmann MA, Dush MK, Martin GR: Rapid production of full-length CDNAs from rare transcripts: amplification using a single gene-specific oligonucleotide primer. Proceedings of the National Academy of Sciences USA 1988, 85:8998-9002.

41. Livak KJ, Schmittgen TD: Analysis of relative gene expression data using real-time quantitative PCR and the 2- $\Delta \Delta C T$ method. Methods 2001, 25:402-408.

42. Czechowski T, Stitt M, Altmann T, Udvardi MK, Scheible WR: Genome-wide identification and testing of superior reference genes for transcript normalization in Arabidopsis. Plant Physiology 2005, 139:5-17.

doi:10.1186/1472-6750-11-36

Cite this article as: Yeoh et al: Developing a method for customized induction of flowering. BMC Biotechnology 2011 11:36.

\section{Submit your next manuscript to BioMed Central and take full advantage of:}

- Convenient online submission

- Thorough peer review

- No space constraints or color figure charges

- Immediate publication on acceptance

- Inclusion in PubMed, CAS, Scopus and Google Scholar

- Research which is freely available for redistribution

Submit your manuscript at www.biomedcentral.com/submit 